\title{
Terrain glissant
}

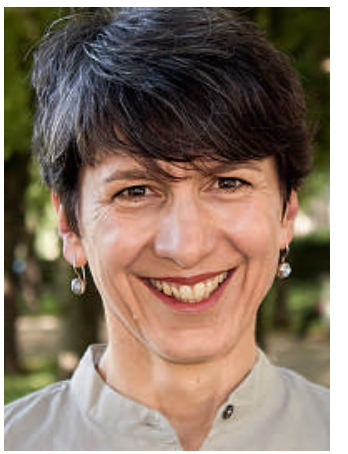

Anna Sax
Pouvez-vous imaginer que des hommes organisent un «PSA Charity Walk» et manifestent avec des milliers de ballons bleus clairs pour plus de solidarité envers les patients atteints d'un cancer de la prostate? Les femmes le font bien, avec des ballons roses bien sûr. En septembre se tiendra comme chaque année en Suisse aussi le «Pink Ribbon Charity Walk», organisé et parrainé par des opérateurs de téléphonie mobile, des fabricants de lingerie, des marques de mode, de bijoux et de cosmétiques. Les organisatrices récoltent de l'argent pour le compte de la Ligue contre le cancer et militent pour attirer l'attention sur le cancer du sein et les programmes de dépistage. L'affiche de la campagne offerte par la marque de cosmétiques Estée Lauder n'a rien à voir avec le cancer hormis peut-être la maigreur des femmes qui y figurent et qui pourrait $\mathrm{y}$ faire penser.

Je n'y peux rien, mais dans ce genre de manifestation appelant à la solidarité envers les personnes atteintes de cancer, je me sens mal à l'aise. Ces actions se veulent émotionnelles alors qu'elles ne sont en premier lieu qu'une manière de faire de l'argent. Je préfèrerais que l'on me fournisse des éléments concrets et des données statistiques vulgarisées comme le font notamment depuis plusieurs années la Fédération suisse des patients et la Fondation pour la protection des consommateurs. Mais cela n'est guère séduisant et les riches partenaires de parrainage manquent à l'appel.

Quiconque se base sur des données statistiques et met de côté les destins individuels s'aventure en terrain glissant. Les différends sur le test PSA et les débats sur les mammographies de dépistage en sont

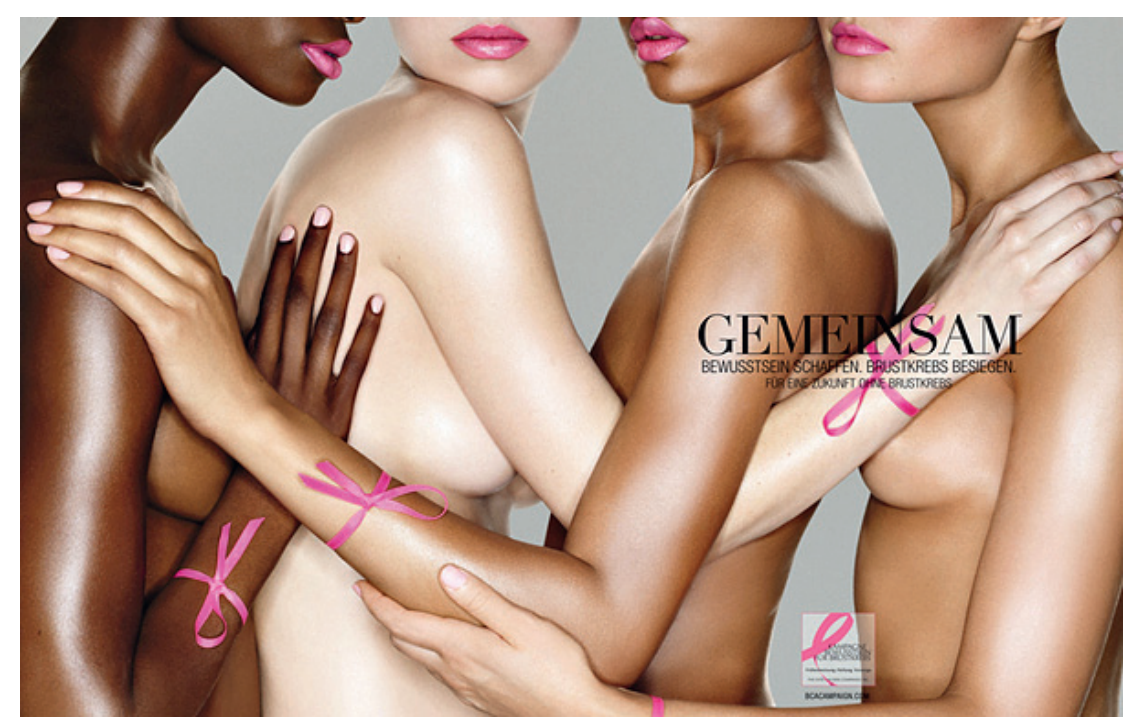

la preuve. Pour les défenseurs des tests de dépistage, ces contrôles doivent se faire à large échelle, car toute anomalie détectée de façon précoce est un succès, et l'éradication sans délai des cellules malignes peut sauver des vies. Et quand il s'agit de sauver la vie d'un homme ou d'une femme, on ne compte pas, on agit. Tout autre comportement ne serait pas éthique. Le calcul des statisticiens qui se basent sur des preuves est plus brutal: les mammographies de dépistage permettent certes de sauver une vie sur 1000, mais un diagnostic sur 10 est faux. Or quel est le plus grand mal? Vaut-il vraiment la peine, pour une femme sauvée, d'en apeurer une centaine d'autres? A-t-on le droit de mettre en rapport les décès potentiellement évitables avec les coûts de surdiagnostic et de surtraitement comme le fait le Swiss Medical Board dans son rapport sur le test PSA?

Garder la vue d'ensemble représente un véritable défi et pas seulement pour les personnes qui ne connaissent rien à la médecine. Les professionnels de la santé sont eux aussi étonnamment mal informés des bases statistiques pour ou contre les programmes de dépistage à large échelle. Selon une étude allemande, seule une minorité des spécialistes interrogés était en mesure d'interpréter correctement la différence entre une réduction de risque absolue et relative. Cela serait notamment dû au fait que les résultats des études publiées dans des revues scientifiques manquent souvent de clarté [1]. Or je suis plutôt d'avis que les médecins pensent d'abord en termes éthiques et seulement ensuite en catégories statistiques. Ils sont plus concernés par les vies à sauver que par la relation coût-utilité des programmes de dépistage. La plupart des urologues et des gynécologues recommandent d'ailleurs à leurs patients et patientes de plus de 50 ans de passer un test PSA ou une mammographie. Il n'est donc guère étonnant que la population revendique des examens préventifs remboursés par l'assurance-maladie pour pouvoir croire en une présumée sécurité.

Reste à espérer que le Swiss Medical Board se penchera prochainement sur les mammographies de dépistage et suscitera ainsi un débat aussi animé que celui sur le test PSA. Il sera ainsi possible de prendre des décisions éclairées.

Anna Sax*

1 Wegwarth O et al. Zu Risiken und Nebenwirkungen ... Wie informiert sind Ärzte und Patienten? Dans: Von Kluse $\mathrm{N}$ et al (ed.). Informiert und selbstbestimmt. Der mündige Bürger als mündiger Patient. Baden-Baden: Nomos-Verlag; 2009. pp. 123 à 138. 